\title{
Vehicular Sensing Networks in a Smart City: Principles, Technologies and Applications
}

\author{
Jingjing Wang, Student Member, IEEE, Chunxiao Jiang, Senior Member, IEEE, \\ Kai Zhang, Tony Q. S. Quek, Senior Member, IEEE, Yong Ren, Senior \\ Member, IEEE, and Lajos Hanzo, Fellow, IEEE
}

\begin{abstract}
Given the escalating population across the globe, it has become paramount to construct smart cities, aiming for improving the management of urban flows relying on efficient information and communication technologies (ICT). Vehicular sensing networks (VSNs) play a critical role in maintaining the efficient operation of smart cities. Naturally, there are numerous challenges to be solved before the wide-spread introduction of VSNs, including the conception of an accurate topological analysis method as well as of a beneficial cooperation mechanism during the process of city-wide information sharing. Hence, in this paper, we construct a VSNs-aided smart city model and appraise a range of intelligent applications in terms of both public services as well as of urban flow management. Then, the information source selection algorithm of a complex network and a reinforcement learning based city information sharing mechanism are considered, complemented by a range of open challenges.
\end{abstract}

\section{Index Terms}

Smart city, vehicular sensing network (VSN), topological analysis, information sharing mechanism.

J. Wang, C. Jiang, K. Zhang and Y. Ren are with the Department of Electronic Engineering, Tsinghua University, Beijing, 100084, China. E-mail: chinaeephd@gmail.com, kaizhangee@gmail.com, \{jchx, reny\}@tsinghua.edu.cn.

T. Q. S. Quek is with Singapore University of Technology and Design, 20 Dover Drive, Singapore 138682. Email: tonyquek@sutd.edu.sg.

L. Hanzo is with the School of Electronic and Computer Science, University of Southampton, Southampton, SO17 1BJ U.K. Email: 1h@ecs.soton.ac.uk.

L. Hanzo would like to gratefully acknowledge the financial support of the European Research Council's Advance Fellow Grant Beam Me Up as well as that of the Royal Society's Wolfson Research Merit Award.

IEEE Wireless Communications Magazine, 2017. Since this is a magazine paper, it has no research data. 


\section{INTRODUCTION}

The 'smart city' concept was proposed both for resolving bottlenecks during urbanization, as well as for supporting sustainable urban development. Relying on efficient information and communication technologies (ICT), a smart city is capable of sensing, analyzing, and integrating the critical information in a city's operation and development, including public security information, industrial activities, social public services, etc. In view of the emergence and rapid development of the Internet of Things (IoT) and cloud computing [1], a smart city is beneficial in terms of enhancing the quality, performance and interactivity of urban services, of reducing costs and resource wastage, as well as of improving the government's management capability. For example, San Diego in the USA was shaped to become a 'City of the Future' and Canada proposed the 'Smart Capital' concept for Ottawa. Moreover, Singapore intended to construct an 'Intelligent Island' and constructing 'Smart Shanghai' was targeted by the government of China.

In the construction of a smart city, an intelligent transportation system (ITS) may be deemed to be the most crucial function given the proliferation of vehicles and the constant expansion of road-networks [2]. During the last decades, the total number of vehicles in the city increased faster than the population. Therefore, ITS is beneficial both in terms of increasing the efficiency of traffic management, as well as of providing compelling applications for a smart city. Based on the maturing technologies in the vehicular ad hoc networks (VANETs), vehicular sensing networks (VSNs) may be invoked both for vehicle-to-vehicle (V2V) communications and vehicleto-infrastructure (V2I) interactions, as well as for sensing, transmitting and integrating important information related to a city's operation for preventing traffic jams.

Relying on the cooperation and coordination among vehicles, diverse sources of information may be sensed, transmitted and integrated in the context of VSNs. However, in view of the impact of different road conditions and of sudden traffic accidents, VSNs have irregularly fluctuating dynamic topological structures, which gives rise to grave communication challenges, often leading to a high bit error rate (BER) and long delays. Furthermore, a small number of malicious vehicle-nodes may report false information for the sake of unfair private benefits, which threatens the city's information security in VSNs. These challenges gave us the motivation to conceive this article on the application and key technologies of VSNs in a smart city. Specifically, 
we listed several intelligent applications in a smart city relying on the basic architecture of VSNs. Moreover, based on the theory of complex networks, a new topology analysis method tested on a real dataset of taxi GPS traces in Beijing was proposed, including an optimal information source selection technique. We also conceived the theory of sharing genuine information in VSNs.

The remainder of the article is outlined as follows. The VSNs' architectures, key technologies and applications in a smart city are introduced in Section II. Optimal information source selection designed for VSNs and the associated critical research challenges are discussed in Section III. In Section IV, our technique of sharing genuine information based on reinforcement learning is investigated, followed by a range of future work ideas and our conclusions in Section V.

\section{Vehicular Sensing Networks in a Smart City}

The smart city concept may bring about dramatic changes in our urban life style, as well as in the way smart cities are managed. VSNs play a critical part both in constructing an intelligent transportation system (ITS), and in providing comprehensive information services for the smart city.

\section{A. VSNs: Architectures and Applications in a Smart City}

Fig. 1 shows the architecture of VSNs constructed for the smart city. Specifically, the vehicles glean information from a multiplicity of city operations, and provide broadcast services with the aid of V2V or V2I communication based on a certain routing strategy. Moreover, relying on the access networks or other hot spots, important information can also be shared on the Internet, while the passengers are capable both of surfing the Internet and of enjoying rich multimedia services. Based on this information, both efficient traffic management and long-term urban planning becomes possible [3].

Based on the VSN, as well as on the intelligent wireless information infrastructure and sharing, smart and prompt sensing, delivery and processing of the data becomes feasible. In the following, we focus our attention on a number of compelling applications of VSNs, which are not necessarily limited to smart city scenarios.

1) Traffic Management: Traffic congestion is an acute problem for urban administrators, especially in the metropoles of the globe. Traditional traffic management policies, such as oddeven day vehicle bans, license plate quota, etc., which may relieve the traffic congestions to 


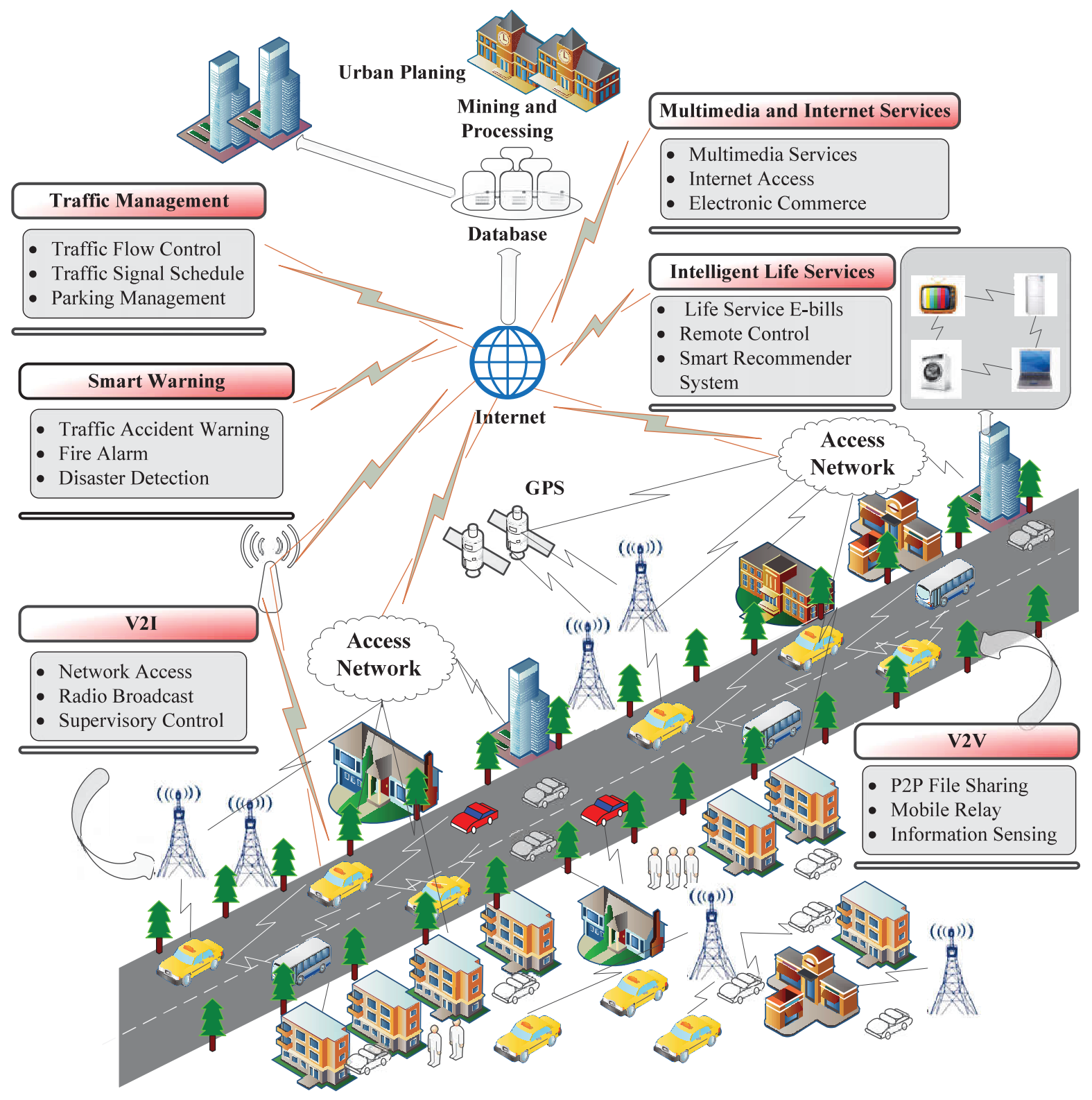

Fig. 1. The architecture and applications of VSNs in a smart city.

some extent fail to take into account the road-network's conditions, nor do they consider the urban population distribution and the peak/off-peak time factor. An effective solution to traffic management relies on the information interaction and coordination in the VSNs, which is capable of planning and recommending optimal driving routes, maintaining a reasonable traffic flow, as well as providing constructive feedback for future urban road-network design. With the aid of smart vehicle sensors as well as beneficial information processing methods in the VSNs, an 
intelligent urban transportation monitoring and management mechanism can be constructed [4].

Specifically, the traffic flow prediction mechanism is capable of relieving the roads having a heavy traffic and/or predicting the peak-traffic time relying on historical observation data as well as on real-time GPS location information, which is beneficial for recommending routes for each vehicle. Moreover, the traffic light scheduling management may be invoked to control the traffic flow in order to approach the maximum network throughput. The vehicle speed management system broadcasts the notification of speed limits, which aims both for ensuring the traffic safety as well as for exploiting the road conditions. Furthermore, parking management may be conducted for informing the drivers of the available parking bays and their up-to-date prices.

2) Smart Warning: Accidents are a common occurrence in daily urban life. An early warning is capable of minimizing the loss of the people's lives and damage to their properties both in case of traffic accidents and in other emergency situations, such as fire hazard, water pipe leakage, etc [5]. Moreover, it is beneficial for preventing secondary accidents as well. Relying on the flock of vehicles and on their widely distributed locations in a city, VSNs provide an ideal replacement for the traditional urban accident-warning anytime and anywhere. Given the information sharing mechanism of VSNs, when and where a traffic accident has happened will be spread promptly. The timely and effective accident-warning is conducive in terms of assisting the drivers' decision-making in order to avoid traffic jams. Furthermore, a potential fire or a water/gas leakage may be perceived by the intelligent sensor of a vehicle, which immediately raises the alarm to the city's command-and-control center, where further measures will be taken.

3) Multimedia and Internet Services: The VSN can be viewed as a mobile ad hoc network. Relying on the emerging device-to-device (D2D) and device-to-infrastructure (D2I) communication modes, as well as on the cellular technology, VSNs play a bridging role in the construction of a metropolitan area network (MAN), or even of a nation-wide super-WiFi system. The dense yet widely-distributed vehicles of a VSNs significantly increase the number of wireless relays and access points available for the MAN, which is capable of providing both stable multimedia services as well as high-rate Internet services [6]. These mobile vehicles allow the intelligent MAN both to reduce its dependence on fixed base stations, as well as to improve the other Internet access.

4) Reliability of Intelligent Services: In addition to collecting traffic information, road conditions and any other sensory information an ITS needs, relying on sharing genuine 
information rather than bluffing and spoofing, VSNs can be used for sensing and handling the genuine 'water, electricity and gas billing information' by a car driving past each property. Specifically, each vehicle may be assigned a unique ID number, which is bound to specific utility bills. Based on the relevant facilities located along the road-network, the vehicles are capable of reading the water, gas and electricity meters and reporting them automatically. Hence, no one will have to worry about the extra fine for an overdue payment. Another intelligent utility provided by VSNs in a smart city is the information recommendation system, provided that the VSNs are reliably linked to intelligent homes and personal information system. Relying on advanced data-mining and machine-learning aided analysis, the driver will receive recommendations from VSNs based on his/her personal taste.

5) Urban Planning: Urban planning is not only for policy-makers and managers, it affects the residents' quality of life as well, since beneficial urban planning offers convenience and comfort for the population. Efficient urban planning supports the convenient movement of the population, as well as of goods and other resources. In a nutshell, VSNs are capable of collecting relevant urban information and of feeding it back to the policy-makers. Aided by the VSNs, road-network layout planning and infrastructure construction becomes more efficient.

\section{B. Key Technologies of VSNS}

As mentioned above, VSNs play a critical role in constructing a smart city in numerous ways. To make the pioneering smart city applications a reality, VSNs face numerous open challenges. Efficient key technologies and protocols, such as the continuous air-interface conceived for longand medium- range (CALM) enacted by the international organization for standardization (ISO), as well as the car-to-car network architecture (C2CNet) designed by the car-to-car communication consortium (C2C-CC), and the IEEE 1609 protocol, were discussed in [7] for constructing a large-scale, mobile, ad hoc vehicular network. Previous studies of the key technologies of VSNs can be summarized as follows.

- Wireless Channel Characteristics: Limited bandwidth, high Doppler shift and building occlusions are the main challenges facing the VSNs of a smart city.

- Mobility and Dynamic Topology: Another characteristic of VSNs is the unpredictable mobility of each vehicle, which leads to a regionally inhomogeneous but globally stable 
network topology. Detailed analysis of the dynamic VSN topology assists the research of tele-traffic routing strategy.

- Efficient Routing Strategy: Providing an efficient tele-traffic routing strategy is beneficial for optimizing the allocation of bandwidth as well as for reducing the risk of network congestion.

- Congestion Control: An appropriate transmission congestion control scheme is capable of reducing the communication blocking probabilities, hence improving the network throughput.

- Security Mechanism: Relying on VSNs, a large amount of personal information has to be acquired and shared in support of smart city services. In order to prevent malicious information interception, reliable security mechanism has to be designed.

Table I provides a brief summary of the most representative previous studies on key technologies of VSNs.

\section{Complex Network Topology-BASEd Optimal Information Source Selection FOR VSNS}

In subject area of complex network topology, we are dealing with a graph-based model of realistic systems exhibiting more random features than lattices, for example. In order to support the above compelling applications of VSNs in a smart city mentioned in Section II-A, we have to consider a number of key technologies, such as VSN's topology and the selection mechanisms of the different information sources as well as their transmission and processing. Fig. 2 depicts two application scenarios in a smart city, including a fire-warning and smart gas meter-reading, where identifying the optimal information source selection mechanism is beneficial in terms of prompt information broadcasting with the aid of the limited bandwidth resources of VSNs. In this section, we commence with an overview of the prevalent vehicular topology. Then, based on this we will introduce our latest research contributions.

\section{A. Topology Analysis of VSNs: An Overview}

At the time of writing, a variety of network topology analysis methods have been proposed in the literature for VSNs. Generally, the topological structures of VSNs rely on a deterministic road topology and on a stochastic vehicular topology. Specifically, the road topology is unlikely 
TABLE I

BRIEF DESCRIPTIONS OF RELATED KEY TECHNOLOGIES OF VSNS.

\begin{tabular}{|c|c|c|}
\hline$\overline{\overline{\text { Key Issues }}}$ & $\overline{\text { Related study }}$ & $\overline{\text { Brief Descriptions }}$ \\
\hline \multirow{4}{*}{ Channel } & $\begin{array}{l}\text { - Non-geometry Based Model, } \\
\text { I. Sen, } 2008\end{array}$ & $\begin{array}{l}\text { - Channel measurement and modeling was proposed for } \mathrm{V} 2 \mathrm{~V} \\
\text { scenarios. }\end{array}$ \\
\hline & $\begin{array}{l}\text { - Geometry Based Stochastic Model } \\
\text { (GSCM), J. Karedal, } 2009\end{array}$ & $\begin{array}{l}\text { - Developed a generic modeling approach for V2V channels } \\
\text { for rural area. }\end{array}$ \\
\hline & $\begin{array}{l}\text {-Geometry-based Efficient Model } \\
\text { (GEM), M. Boban, } 2014\end{array}$ & $\begin{array}{l}\text { - A propagation model based on different link classification, } \\
\text { having large-scale/small-scale signal variation. }\end{array}$ \\
\hline & $\begin{array}{l}\text { - Dynamic Wide-band Directional } \\
\text { Channel Model, R. He, } 2015\end{array}$ & $\begin{array}{l}\text { - Proposed an empirical dynamic V2V model for time-variant } \\
\text { V2V channels relying on dynamic scatterers. }\end{array}$ \\
\hline \multirow{4}{*}{ Mobility } & $\begin{array}{l}\text { - Connectivity Dynamics Analysis of } \\
\text { Vehicular Mobility, M. Fiore, } 2008\end{array}$ & $\begin{array}{l}\text { - Revealed the relationship between network topology and } \\
\text { vehicular mobility. }\end{array}$ \\
\hline & $\begin{array}{l}\text { - Mobility Model Pattern Generator } \\
\text { CityMob, F. Martinez, } 2008\end{array}$ & $\begin{array}{l}\text { - Built an urban vehicular mobility model in order to generate } \\
\text { accurate urban mobility scenarios. }\end{array}$ \\
\hline & $\begin{array}{l}\text { - Network Mobility (NEMO) Manage- } \\
\text { ment for VANET, Y. Chen, } 2010\end{array}$ & $\begin{array}{l}\text { - Proposed a network mobility management protocol for VANET } \\
\text {,having a reduced mobility handoff time and packet loss rate. }\end{array}$ \\
\hline & $\begin{array}{l}\text { - Obstacle-based Vehicle Mobility } \\
\text { Channel Mode, N. Akhtar, } 2015\end{array}$ & $\begin{array}{l}\text { - Incorporated a more realistic obstacle-based channel model } \\
\text { into the analysis of VANET topology characteristics. }\end{array}$ \\
\hline \multirow{4}{*}{ Routing } & $\begin{array}{l}\text { - Intersection-based Geographical Ro- } \\
\text { uting Protocol (IGRP), H. Saleet, } 2011\end{array}$ & $\begin{array}{l}\text { - Relying on a selection of road intersections, Saleet modeled } \\
\text { the QoS routing problem as a constrained optimization. }\end{array}$ \\
\hline & $\begin{array}{l}\text { - Intelligent Optimized Link State } \\
\text { Routing (IOLSR), J. Toutouh, } 2012\end{array}$ & $\begin{array}{l}\text { - Proposed the IOLSR for automatically obtaining the configu- } \\
\text { ration that best fits the specific characteristics of VANETs. }\end{array}$ \\
\hline & $\begin{array}{l}\text { - Hybrid Bio-inspired Bee Swarm } \\
\text { Routing (HyBR), S. Bitam, } 2013\end{array}$ & $\begin{array}{l}\text { - A uni-cast and multi-path routing protocol was proposed for } \\
\text { urban and rural scenarios having little delay and overheads. }\end{array}$ \\
\hline & $\begin{array}{l}\text { - Multihop-Authenticated Proxy Mobile } \\
\text { IP (MA-PMIP), S. Cspedes, } 2013\end{array}$ & $\begin{array}{l}\text { - Studied the secure handover of IP services in an asymme- } \\
\text { tric VANET relying on location and traffic information. }\end{array}$ \\
\hline \multirow{4}{*}{ Transmission } & $\begin{array}{l}\text { - Adaptive Inter-vehicle Communication } \\
\text { Control, C. Huang, } 2010\end{array}$ & $\begin{array}{l}\text { - Proposed a transmission control protocol in order to adapt both } \\
\text { the communication rate and the power based on the VANET. }\end{array}$ \\
\hline & $\begin{array}{l}\text { - Encounter Transfer Protocol } \\
\text { (ETP), B. Yu, } 2011\end{array}$ & $\begin{array}{l}\text { - Promptly adapts to fast-changing channel conditions and } \\
\text { fully utilize the precious link duration as vehicles encounter. }\end{array}$ \\
\hline & $\begin{array}{l}\text { - Priority-Based Congestion Control } \\
\text { Algorithm, L. Tung, } 2013\end{array}$ & $\begin{array}{l}\text { - A prioritization scheme was proposed to exploit the limited } \\
\text { bandwidth for cross-traffic assistance. }\end{array}$ \\
\hline & $\begin{array}{l}\text { - Linear Message Rate Integrated } \\
\text { Control (LIMERIC), G. Bansal, } 2013\end{array}$ & $\begin{array}{l}\text { - Defined an adaptive congestion control algorithm applied to } \\
\text { the message rate of devices in vehicular environment. }\end{array}$ \\
\hline \multirow{4}{*}{ Security } & $\begin{array}{l}\text { - Cryptography-based Key Distribution } \\
\text { Protocol, H Schweppe, } 2011\end{array}$ & $\begin{array}{l}\text { - Introduced a communication security architecture for Car2X } \\
\text { and a secure transport protocol for in-vehicle communication. }\end{array}$ \\
\hline & $\begin{array}{l}\text { - Data Flow Tracking in Automotive On- } \\
\text { board Network, H. Schweppe, } 2012\end{array}$ & $\begin{array}{l}\text { - A security framework was proposed for monitoring data flows } \\
\text { in order to achieve improved security. }\end{array}$ \\
\hline & $\begin{array}{l}\text { - Signature-based Authentication for } \\
\text { DoS Attacks, L. He, } 2012\end{array}$ & $\begin{array}{l}\text { - Relying on the so-called one-way hash chain and group rekeying } \\
\text { scheme, pre-authentication was conceived for preventing attacks. }\end{array}$ \\
\hline & $\begin{array}{l}\text { - Trust Evaluation-based Security } \\
\text { Authentication, A. Zhou, } 2015\end{array}$ & $\begin{array}{l}\text { - Proposed a security authentication method based on trust } \\
\text { evaluation, which is quantified by recommendation trust vectors. }\end{array}$ \\
\hline
\end{tabular}

to change in the short term, once it has been completed in a city. However, extreme weather conditions causing land-slides or flooding as well as the construction of new roads may influence the long-term road-topology. Moreover, resurfacing the roads, burst gas or water-pipes as well as traffic jams may lead to more short-term changes of the road-network's connectivity. By contrast, given the multiplicity of mobile vehicles in a city, the vehicular topology is subject to near-instantaneous change. In order to characterize the stochastic vehicular topology, we 


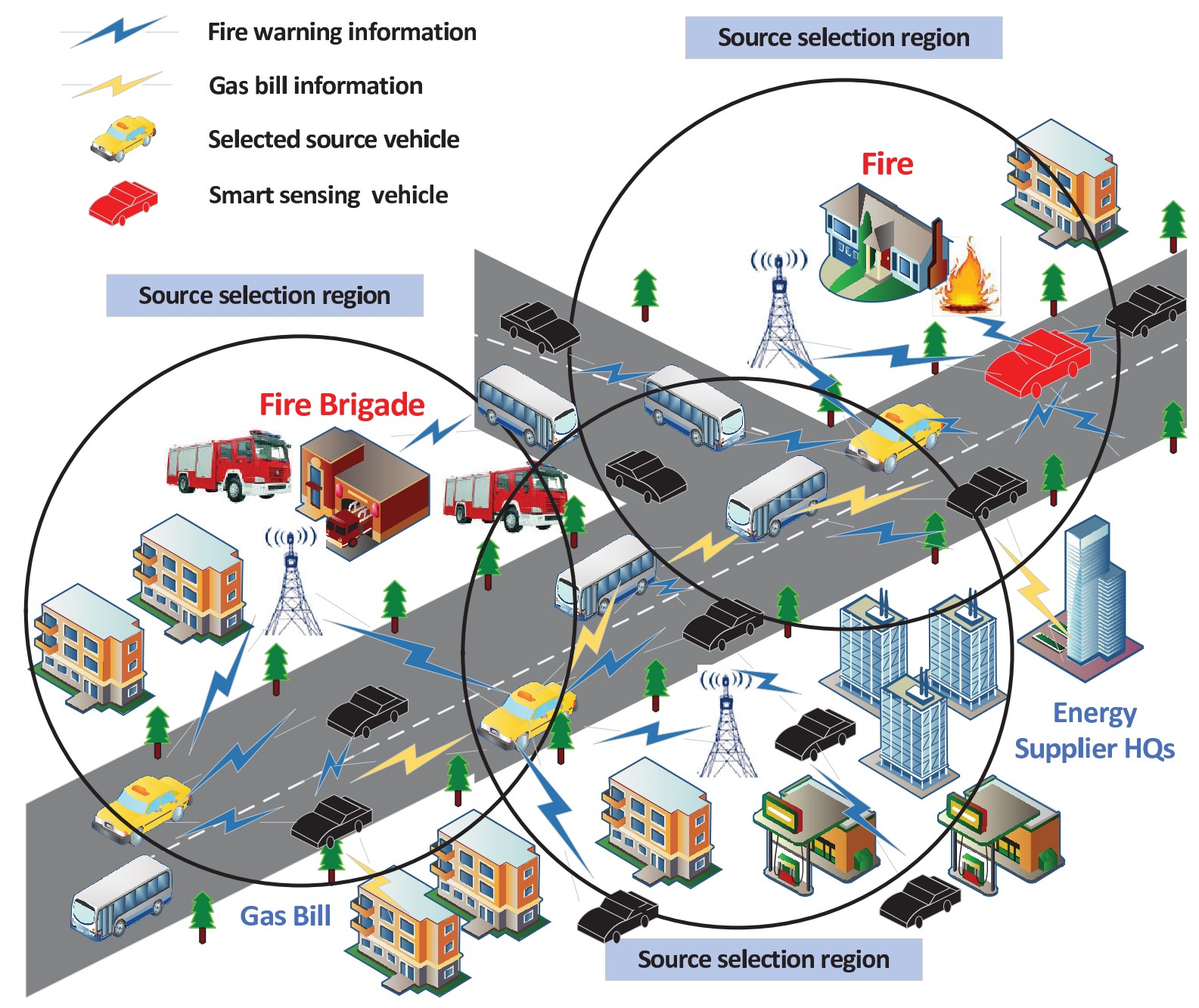

Fig. 2. Two application scenarios of VSNs in a smart city. (Fire warning and smart gas meter-reading)

discretize the time-axis into time-slots. In each time slot, the vehicular topology is deemed to be a static.

The topology analysis of the VSNs plays a critical role in characterizing the inherent relationship between each pair of communication nodes in order to design beneficial communication mechanisms, because an inaccurate representation of the VSNs' topology result in a poor performance. Hence, it is necessary to perform an accurate topological analysis in order to construct an accurate model of VSNs. Moreover, we are able to model the vehicular network topology both by relying on theoretical derivations, as well as based on statistical analysis and software simulations. Specifically, Mahmoud et al. [8] conceived a vehicular traffic flow model and the corresponding link reliability model for characterizing their mobility within a vehicular 
network. As a further development in the field, Uppoor et al. [9] designed a realistic synthetic database trained by recording 24 hours of car traffic in a $400-\mathrm{km}^{2}$ region around the city of Köln, in Germany. Relying on this database, the networks' connectivity was characterized in comparison to several other mobility traces commonly used in vehicular network simulation. In [10], Ferreira et al. described several sophisticated traffic simulators, which relied either on car-following models or cellular automata models, such as the Simulation of Urban MObility (SUMO), the TRansportation ANalysis and SIMulation System (TRANSIMS), the Development of Inter-VEhicular Reliable Telematics (DIVERT), etc. Relying on realistic road topologies and a rich set of vehicular parameters, these simulators are capable of generating realistic vehicular mobility models.

Nevertheless, both the theory-based as well as the simulator-based modeling methods only concentrate on a basic characterisation of the road-network. However, they are typically oblivious of human factors, such as the drivers' habits, or of the dominant direction of population movement at certain periods of the working day, albeit these have a substantial influence both on the network topology as well as on its dynamic evolution. In contrast to the above-mentioned theorybased and simulator-based modeling methods, vehicular network models relying on a statistical database may be characteristic of the practical situation. However, a region-specific database is not universally suitable for all regions and cities. Having said this, it is impractical to construct a separate database for every city. Accordingly, we have to explore a vehicular network modeling method capable of characterizing both the objective conditions as well as the subjective human factors, which is of pivotal significance during the design of VSNs, optimization and management in a smart city.

\section{B. Optimal Information Source Selection: Based on a Complex Network Perspective}

In this subsection, we focus our attention on the topological analysis of VSNs in terms of a complex network theory perspective. Based on this, an optimal information source selection algorithm is proposed. First, we constructed a vehicular network model based on a real-world dataset, which was recorded by storing the GPS positions of taxies in Beijing (longitude ranging from 116.25 to 116.55 and latitude ranging from 39.8 to 40.05). As shown in Fig. 3 (a), the taxi position distribution clearly reflected the planning of the urban structure and distinguished the downtown and suburban areas of Beijing. Secondly, we included the edges between the pair 


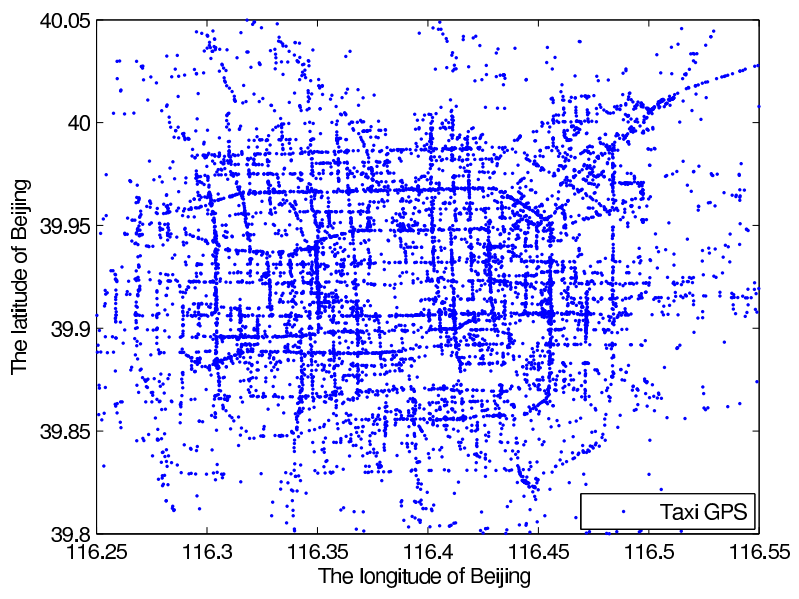

(a) Taxi GPS distribution in Beijing city

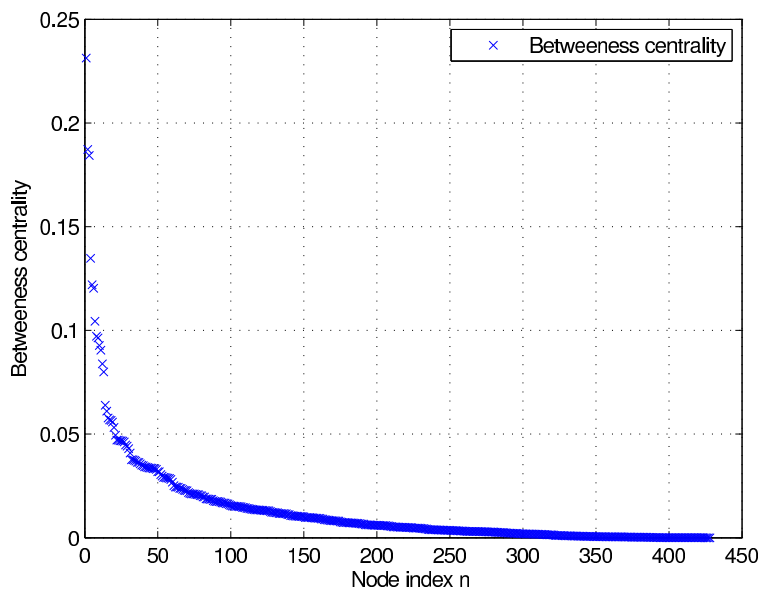

(c) Betweenness centrality

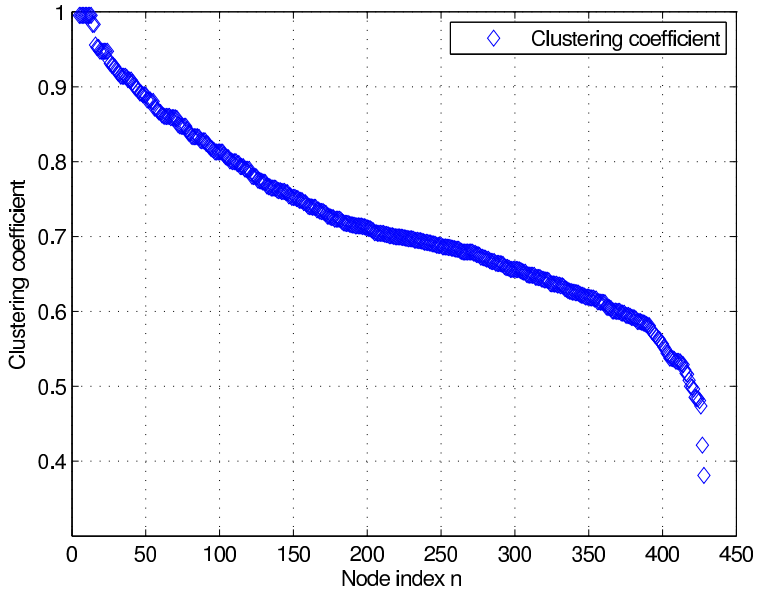

(b) Clustering coefficient

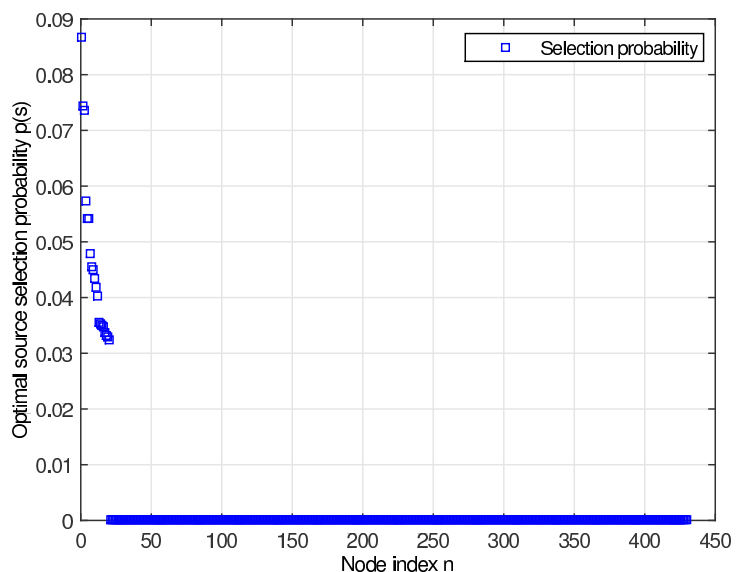

(d) Source selection probability distribution $p(s)$

Fig. 3. Complex network topology-based optimal information source selection for VSNs.

of discrete points representing the vehicles according to the communication distances among them. Explicitly, two vehicles were connected by an edge, when their distance was confined within the maximum communication radius $r$. In our work, we adopted an empirical maximum communication radius of $r=500 \mathrm{~m}$ and considered a reduced-size subregion determined by the longitude ranging from 116.32 to 116.39 and latitude ranging from 39.96 to 39.985. Below, further complex-network based topological discussions are presented.

Again, a complex network constituted by a number of agents, which typically exhibits the small-world phenomenon, indicating that each agent may be reached by any other agent within six hops. In the following, we determined several complex network parameters relying on our unweighed and undirected vehicular network. 
- Clustering Coefficient: The ability of neighbors to communicate with each other is characterized by the clustering coefficient, which reflects the tightness of the network. Fig. 3 (b) shows the clustering coefficient of each vehicle in a descending order. The average value of the clustering coefficients is $C=0.7725$, which physically means that the vehicular network has a close-knit community structure.

- Betweenness Centrality: Generally, a vehicle having a large node degree plays a critical role during the communication. However, under some circumstances, a vehicle having a small degree may also act as a bridging node. Therefore, the associated betweenness centrality, shown in Fig. 3 (c) is defined as a measure of importance of the node in the network, which is defined as the number of shortest paths leading from all vertices to all others that pass through the specific node considered. Relying on the power-law based distribution of betweenness centrality, we can conclude that only a few vehicles of the network constitute a bottleneck during a communication session. It is critical for us to pay more attention to the key vehicles at crossroads, transportation junctions or to the ones playing the role of bridges than to the boundary vehicles.

- Average Path Length: Average path length (APL) is proposed as the average number of hops along the shortest paths for all possible pairs of the network nodes. The APL of our vehicular network is $L=6.7337$.

Hence, our vehicular network obeys the small-world property, while exhibiting a high degree of clustering, but a short average path length associated with $C=0.7725$ and $L=6.7337$. Relying on the above characteristics of our complex network, we constructed a weight matrix characterizing each edge in the vehicular network, which represented the communication performances among vehicles as well as between the vehicles and the infrastructure. Specifically, the weight of each edge was determined by the communication distance, channel's fading model, by the signal to noise ratio, and by the the vehicles' parameters. The weight matrix was synonymously referred to the communication impedance. Therefore, a weighed and undirected topological model $G$ was formulated as $G=<V, E, W>$, where $V, E$ and $W$ represented the set of vehicle nodes, the set of communication links, as well as the weight matrix, respectively.

In the VSNs, the specific location of the information sources directly influences the communication efficiency achieved, and the communication overhead imposed, especially in an information broadcast scenario. A compelling optimization criterion for an information source 
selection algorithm is to maximize the attainable network capacity of the VSN considered. Relying on our complex-network based vehicular topology, we characterized the source vehicles in terms of a probability distribution $p(S)$, while assuming that the destination vehicles were uniformly distributed and that they were independently selected. Hence, the total network capacity $R_{c}$ can be formulated as a function of the communication impedances. Moreover, maximizing the network capacity $R_{c}$ was shown to be equivalent to a min-max optimization problem, which can be reduced to a linear programming problem [11]. Fig. 3 (d) presents the source selection probability distribution of each vehicle relying on our topological model of Fig. 3 (a). Accordingly, only a few vehicles acted as information source nodes in the vehicular networks considered. In other words, the information source vehicles are within limited distance, corresponding to a limited number of hops.

\section{City Information Sharing Theory FOR VSNs}

Wireless VSNs support a multitude of intelligent applications in a smart city. Information dissemination/sharing among vehicles is one of the fundamental benefits of vehicular networks, which supports a diverse range of road traffic services as well as other scenarios. Returning to the pair of scenarios shown in Fig. 2, the cooperation of vehicles is capable of ensuring an efficient spreading of city information. However, the provision of manipulated information by selfish vehicles may lead to potentially avoidable traffic jams, for example. Similarly, the utility meter-reading may be decyphered by eavesdroppers and traded during the process of city information sharing by the malicious vehicles. In the following, we focus our attention on the city information dissemination/sharing mechanisms of VSNs.

\section{A. City Information Sharing in VSNs}

Vehicular communications and their support networks have originally been proposed for public safety applications and traffic efficiency enhancements, where the efficiency of information sharing critically relies on the cooperation and coordination of the vehicles as well as on that between the vehicles and the infrastructure [12]. Cenerario et al. [13] constructed an event-related information exchange/sharing protocol of the VSNs. Relying on the short interactions amongst the vehicles to exchange/share information about relevant events, they proposed the concept of encounter probability, in order to decide when an information re-diffusion becomes feasible. 
Given the re-diffusion mechanism, this information exchange/sharing protocol can be applied in a range of vehicular network scenarios, including the quest for parking spaces, the broadcasting of accidents or obstacles along the road ahead, announcing the routing of emergency vehicles, etc. Further applications in a smart city mentioned in Section II-A can be implemented in terms of the in-depth city information sharing mechanisms.

Although most of the vehicles act in a cooperative manner during the information sharing, a small number of selfish or malicious ones may deliberately provide either random or manipulated information for the sake of attaining an unfair priority. In order to make this behaviour more unattractive during the information sharing, Fadul et al. [14] created a trust-management toolkit for identifying and mitigating the compromised actions of the nodes. Relying on Fadul's work [14], a four-module information sharing framework was conceived for constructing a genuine information sharing mechanism, which relies on a monitoring module, a reputationmanagement module, a path-management module and on a trust-management module.

- Monitoring Module: Each vehicle is equipped with a monitoring module in order to both detect the misbehaviour as well as to provide responses to the cooperating vehicles. When detecting a given malicious behavior, the monitoring module activates the reputationmanagement module;

- Reputation-Management Module: The reputation-management module evaluates the vehicle's integrity according to historical records, whilst relying on online learning algorithms. The information received from malicious vehicles whose reputation scores cannot reach a certain threshold, will be forwarded to the path-management module;

- Path-Management Module: The path-management module is in charge of redirecting the information dissemination paths relying on the attributes of the vehicles derived from the report provided by the reputation-management module;

- Trust-Management Module: The trust-management module relies on a threshold. Moreover, it includes a list of both genuine as well as of malicious nodes.

As a further development, Khabazian et al. [15] presented an analytical model for characterizing the performance measures of information sharing in VSNs under the assumption of two priority classes of traffic. Specifically, the distribution of the number of concurrent transmissions was discussed, as well as numerical results were provided along with simulation results in order 

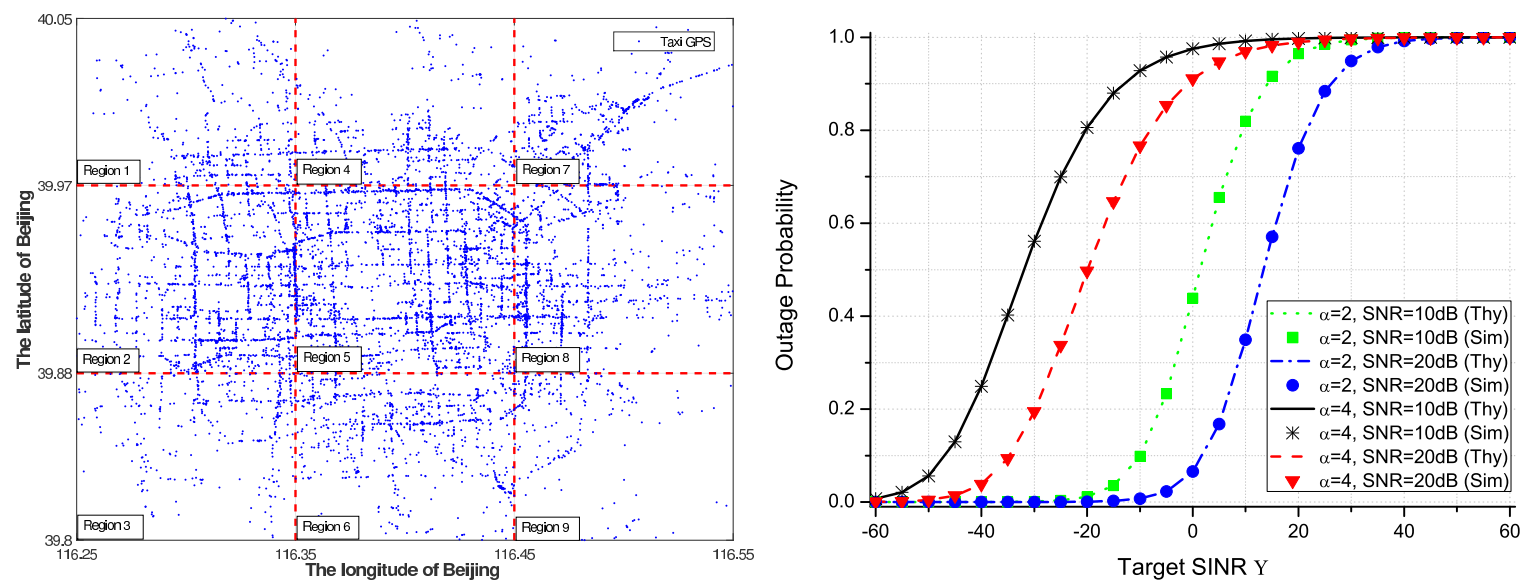

(a) A nine-block separation of Beijing city

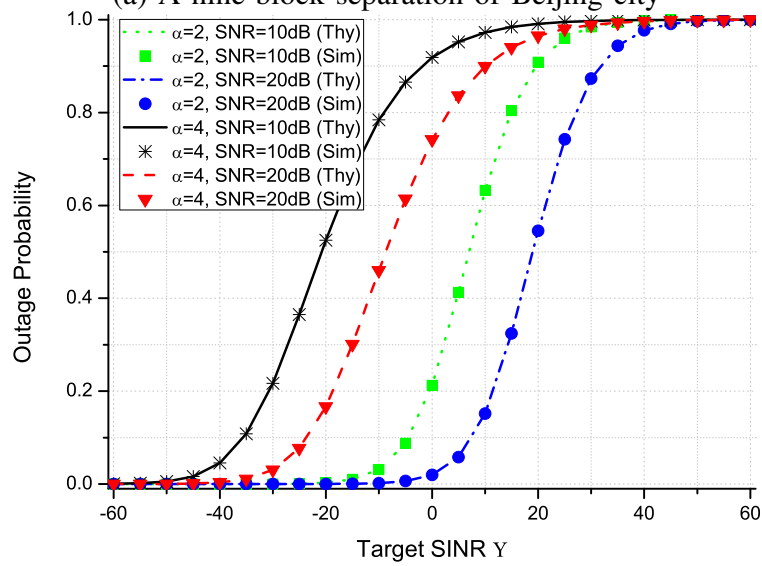

(c) Outage probability of downtown scenario in Region 5

(b) Outage probability of suburban scenario in Region 7

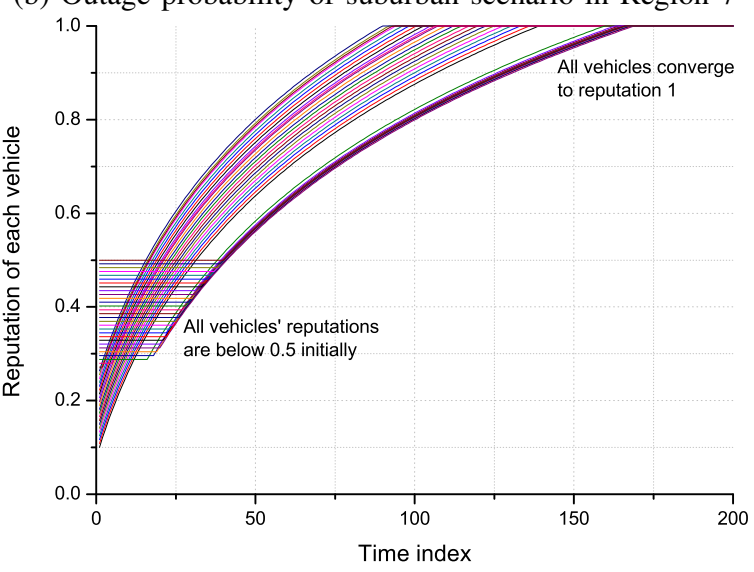

(d) Dynamic reputation of all vehicles

Fig. 4. Simulation results of the mechanism of sharing genuine information.

to confirm the accuracy of the proposed analysis. Moreover, several other performance indicators, such as the packet loss rate, packet transmission distance, end-to-end reliability, throughput, delay were proposed for characterizing the performance of information sharing in VSNs.

\section{B. Sharing Genuine Information with the Aid of Reinforcement Learning}

In this subsection, we focus our attention on designing a specific mechanism relying on reinforcement learning in order to ensure that all vehicles share genuine information. We assume that each vehicle has the capability of acquiring city information and that they are willing to share it with each other in order to make better-informed decisions. Moveover, all the vehicles aim for maximizing their own utility. Relying on the above assumption, we invoke the outage probability of sharing genuine information. We consider both a Nakagami-m fading and a more specific 
highway scenario of a Rayleigh fading channel. Specifically, the outage probability (OP) of a specific vehicle $v_{i}$ is defined as the probability of its signal to interference plus noise ratio (SINR) dipping below a threshold of $\Theta$, which is characterized by the cumulative distribution function (c.d.f) of this vehicle's SINR. Relying on the outage probability, we modeled the vehicles' future utility trend for predicting their future information sharing decisions. Furthermore, a reinforcement learning based reputation evaluation mechanism was designed. Similar to the wellestablished human social networks, each vehicle of a VSN has a reputation value depending on its past behavior, which is also likely to govern its future behavior, when sharing information with others. Given the above basic assumptions, the information sharing process was modeled as a game, where the payoff attained is a function both of the outage probability as well as of the joint strategies. Each vehicle adjusted its information sharing behavior based on the estimation of the other vehicles' reputation and on its accumulated perception.

Simulations were conducted to verify our theoretical analysis and to characterize the proposed schemes, which are based on a real-world dataset consisting of the spatial distribution of Beijing taxis. However, in order to distinguish the downtown and suburban areas, we partitioned Beijing city into nine regions, as shown in Fig. 4 (a). Fig. 4 (b) and (c) portray the outage probability of the suburban and downtown scenarios, respectively, where $\alpha$ is the path-loss coefficient. The simulation results were all consistent with the theoretical results. The outage probability of the downtown scenario was lower than that in the suburban scenario due to the reduced distance between a pair of vehicles as well as owing to the benign Nakagami-m fading channels encountered. Moreover, increasing the path loss coefficient $\alpha$ from 2 to 4 can lead to an increase of the outage probability due to the higher power attenuation of the channel, while increasing of transmission power reduced the outage probability. Fig. 4 (d) presents the dynamic reputation of vehicles during their learning and interaction process, which characterized the city information sharing strategy of each vehicle. Although the vehicles were initially configured to have low reputations below 0.5 , our regime encouraged them to share genuine information, hence gradually achieving a near-unity reputation, which corroborated the high efficiency of our city information sharing mechanism. 


\section{FUTURE WORK AND CONCLUSIONS}

The networked cooperation within VSNs has a vast array of beneficial applications in a smart city. A range of future research ideas related to VSNs designed for a smart city can be summarized as follows:

- It is necessary to construct intelligent standardized protocols for future smart cities in order to integrate heterogeenous VSNs with cellular mobile communication networks, with satellite networks and the wireline based networks.

- The network's capacity has to be quantified and techniques of approaching the VSN's capacity have to be found. Given the dynamically changing network topology, agile network-capacity-approaching resource-allocation and source selection algorithm have to be conceived for these demanding operating conditions.

- The network-security and privacy-protection mechanisms of VSNs, such as fingerprint authentication, data encryption, remote attestation, secure payment, etc. have to be carefully considered, in order to support high-integrity personal services in smart cities.

In a nutshell, several application scenarios of VSNs operating in a smart city have been discussed, followed by an overview of a range of associated technologies. Furthermore, we highlighted the advantages of constructing an accurate topological structure for VSNs. Based on this we proposed an information source selection model in order to improve the attainable network capacity. Finally, the pros and cons of various information sharing solutions were investigated. Indeed, VSNs constitute a promising subject-area of smart city research!

\section{REFERENCES}

[1] J. Jin, J. Gubbi, S. Marusic, and M. Palaniswami, "An information framework for creating a smart city through Internet of things," IEEE Internet of Things Journal, vol. 1, no. 2, pp. 112-121, Apr. 2014.

[2] F.-Y. Wang, "Scanning the issue and beyond: Transportation and mobility transformation for smart cities," IEEE Transactions on Intelligent Transportation Systems, vol. 16, no. 2, pp. 525-533, Apr. 2015.

[3] S. Djahel, R. Doolan, G.-M. Muntean, and J. Murphy, "A communications-oriented perspective on traffic management systems for smart cities: Challenges and innovative approaches," IEEE Communications Surveys \& Tutorials, vol. 17, no. 1, pp. 125-151, Mar. 2015.

[4] R. Du, C. Chen, B. Yang, N. Lu, X. Guan, and X. Shen, "Effective urban traffic monitoring by vehicular sensor networks," IEEE Transactions on Vehicular Technology, vol. 64, no. 1, pp. 273-286, Apr. 2015.

[5] J. Liu, Z. Yang, and I. Stojmenovic, "Receiver consensus: on-time warning delivery for vehicular ad-hoc networks," IEEE transactions on emerging topics in computing, vol. 1, no. 1, pp. 57-68, Jul. 2013. 
[6] L. Sarakis, T. Orphanoudakis, H. C. Leligou, S. Voliotis, and A. Voulkidis, "Providing entertainment app lications in VANET environments," IEEE Wireless Communications, vol. 23, no. 1, pp. 30-37, Feb 2016.

[7] G. Karagiannis, O. Altintas, E. Ekici, G. Heijenk, B. Jarupan, K. Lin, and T. Weil, "Vehicular networking: A survey and tutorial on requirements, architectures, challenges, standards and solutions," IEEE Communications Surveys \& Tutorials, vol. 13, no. 4, pp. 584-616, Jul. 2011.

[8] M. H. Eiza and Q. Ni, "An evolving graph-based reliable routing scheme for VANETs," IEEE Transactions on Vehicular Technology, vol. 62, no. 4, pp. 1493-1504, Feb. 2013.

[9] S. Uppoor, O. Trullols-Cruces, M. Fiore, and J. M. Barcelo-Ordinas, "Generation and analysis of a large-scale urban vehicular mobility dataset," IEEE Transactions on Mobile Computing, vol. 13, no. 5, pp. 1061-1075, Feb. 2014.

[10] M. Ferreira, H. Conceição, R. Fernandes, and O. K. Tonguz, "Stereoscopic aerial photography: An alternative to modelbased urban mobility approaches," in Proceedings of the 6th ACM International Workshop on Vehicular Internetworking, Beijing, China, Sep. 2009, pp. 53-62.

[11] J. Wang, C. Jiang, L. Gao, S. Yu, Z. Han, and Y. Ren, "Complex network theoretical analysis on information dissemination over vehicular networks," in 2016 IEEE International Conference on Communications (ICC), Kuala Lumpur, Malaysia, May 2016.

[12] Y. Cao, T. Jiang, and C. Wang, "Cooperative device-to-device communications in cellular networks," IEEE Wireless Communications, vol. 22, no. 3, pp. 124-129, Jun. 2015.

[13] N. Cenerario, T. Delot, and S. Ilarri, "A content-based dissemination protocol for VANETs: Exploiting the encounter probability," IEEE Transactions on Intelligent Transportation Systems, vol. 12, no. 3, pp. 771-782, Jul. 2011.

[14] J. E. Fadul, K. M. Hopkinson, T. R. Andel, and C. A. Sheffield, "A trust-management toolkit for smart-grid protection systems," IEEE Transactions on Power Delivery, vol. 29, no. 4, pp. 1768-1779, Dec. 2014.

[15] M. Khabazian, S. Aissa, and M. Mehmet-Ali, "Performance modeling of message dissemination in vehicular ad hoc networks with priority," IEEE Journal on Selected Areas in Communications, vol. 29, no. 1, pp. 61-71, Jan. 2011. 